\title{
Metronidazole-induced encephalopathy in a patient with pyogenic spondylitis: a case report
}

\author{
Kazutaka Mizuta ${ }^{1}$, Motoki Sonohata $^{2 *}$ (D), Osamu Nozaki ${ }^{1}$, Tomoki Kobatake ${ }^{1}$, Daisuke Nakayama', \\ Tadatsugu Morimoto ${ }^{2}$ and Masaaki Mawatari
}

\begin{abstract}
Background: Metronidazole is an antimicrobial agent commonly used in the treatment of several protozoal and anaerobic infections. Neurotoxicity associated with metronidazole has been rarely reported, and the incidence of metronidazole-induced encephalopathy is unknown. Therefore, the accurate diagnosis of metronidazole-induced encephalopathy is often difficult because of the rarity of the disease.

Case presentation: An 86-year-old woman suffered from pyogenic spondylitis of the lumbar spine. Parvimonas micra, a gram-positive anaerobic bacterial species and a resident of the flora of the oral cavity, was identified in the biopsy specimens. Oral administration of metronidazole $(1500 \mathrm{mg} /$ day $)$ was initiated. Forty-four days after initiating metronidazole (total intake of $66 \mathrm{~g}$ ), she complained of tingling sensations in the upper limbs. After 4 days, she complained of additional symptoms including sensory disturbance of the tongue, dysarthria, and deglutition disorder. Characteristic brain magnetic resonance imaging findings on T2-weighted fluid-attenuated inversion recovery and diffusion-weighted imaging led to the diagnosis of metronidazole-induced encephalopathy. Metronidazole was discontinued, and her neurological symptoms improved 10 days after discontinuation. At 14 days after discontinuation of oral metronidazole, abnormal findings on diffusion-weighted imaging almost disappeared.

Conclusions: With the possibility of needing to prescribe metronidazole in the orthopedic field for the treatment of various infections, orthopedic surgeons are likely to encounter cases of metronidazole-induced encephalopathy. Thus, they should be able to recognize the condition and its potential complications. With increased awareness, early diagnosis with magnetic resonance imaging and discontinuation of metronidazole may become feasible when such patients are referred. Our report presents a detailed account of such a case, which may help in the early diagnosis and treatment of patients with metronidazole-induced encephalopathy. Furthermore, we recommend that patients treated with metronidazole should undergo careful and constant surveillance after starting antibiotic therapy.
\end{abstract}

Keywords: Metronidazole-induced encephalopathy, Antimicrobial, Complication, Magnetic resonance imaging

\footnotetext{
* Correspondence: epc9719@yahoo.co.jp

${ }^{2}$ Department of Orthopaedic Surgery, Faculty of Medicine, Saga University,

5-1-1 Nabeshima, Saga 849-8501, Japan

Full list of author information is available at the end of the article
}

(c) The Author(s). 2018 Open Access This article is distributed under the terms of the Creative Commons Attribution 4.0 International License (http://creativecommons.org/licenses/by/4.0/), which permits unrestricted use, distribution, and reproduction in any medium, provided you give appropriate credit to the original author(s) and the source, provide a link to the Creative Commons license, and indicate if changes were made. The Creative Commons Public Domain Dedication waiver (http://creativecommons.org/publicdomain/zero/1.0/) applies to the data made available in this article, unless otherwise stated. 


\section{Background}

Metronidazole is an antimicrobial agent commonly used to treat several protozoal and anaerobic infections. Especially, metronidazole is widely used as a standard therapeutic agent for Clostridium difficile-associated infection [1]. It has been prescribed for conditions, such as Crohn's disease, intra-abdominal abscess, Helicobacter pylori infection, hepatic encephalopathy, and recurrent pyogenic cholangitis [2]. Although it is well tolerated in common settings, patients may experience serious neurological side effects with both long- and short-term use. Its side effects include an ataxic gait, peripheral neuropathy, cerebellar dysfunction, dysarthria, vestibulotoxicity, cochleotoxicity, seizures, encephalopathy, and visual impairment [3-9]. Neurotoxicity with metronidazole has been rarely reported, and the incidence of metronidazole-induced encephalopathy (MIE) is unknown. Typical MIE is diagnosed on the basis of abnormal signals on magnetic resonance imaging (MRI) involving the cerebellar dentate nuclei. In addition, many structures, including the corpus callosum, inferior colliculus, midbrain, pons, medulla, and bilateral subcortical white matter, are involved; however, the basal ganglia are less involved [10-15]. In addition, making the appropriate diagnosis is often difficult because of the rarity of the disease in the orthopedic field. Here, we report a rare case of MIE in a patient with pyogenic spondylitis.

\section{Case presentation}

An 86-year-old woman with back pain was referred to our hospital. At the time of presentation, she had no fever and no pain at rest. Roentgenography findings were relatively normal with slight narrowing at L1/2 (Fig. 1a). She had no known drug allergies. She was administered analgesics and was kept under observation. One month after her first visit to our hospital, her back pain worsened, and her temperature was $37.2{ }^{\circ} \mathrm{C}$. No neurological abnormalities were noted in her lower limbs. Laboratory findings at that time were as follows: C-reactive protein, $11.5 \mathrm{mg} / \mathrm{dL}$ (reference, < $0.2 \mathrm{mg} / \mathrm{dL}$ ); white blood cell count, $7970 / \mathrm{mm}^{3}$ (neutrophils, $87.7 \%$; reference, $4000-8000 / \mathrm{mm}^{3}$ ); aspartate aminotransferase, $15 \mathrm{IU} / \mathrm{L}$ (reference, 13-33 IU/L); alanine aminotransferase, 13 IU/L (reference, 6-30 IU/L); alkaline phosphatase, 291 IU/L (reference, 115-359 IU/L); and creatinine, $1.5 \mathrm{mg} / \mathrm{dL}$ (0.4-0.7 mg/dL). Radiographs of the lumbar spine showed collapse of L1 and an absorbable change in the caudal side of L1 (Fig. 1b). Lumbar MRI confirmed the presence of fluid at the L1/2 disc, edema and destruction of the spinal body of $\mathrm{L} 1 / 2$, and lumbar canal stenosis at the level of $\mathrm{L} 1 / 2$ on fat-saturated T2-weighted images (Fig. 1c). She was diagnosed with pyogenic spondylitis of the lumbar spine and underwent biopsy of the L1/2 disc. Parvimonas micra, which is a gram-positive anaerobic bacterial species and a resident of the flora of the oral cavity, was identified in the biopsy specimens. Given that the patient was able to tolerate oral medications and the transfer rate of metronidazole to the blood was similar between oral and intravenous administrations, we decided to administer the medications orally. Oral administration of metronidazole (1500 mg/day) was initiated (Fig. 2). Forty-four days after starting metronidazole (total intake of $66 \mathrm{~g})$, she complained of tingling in the upper limbs.

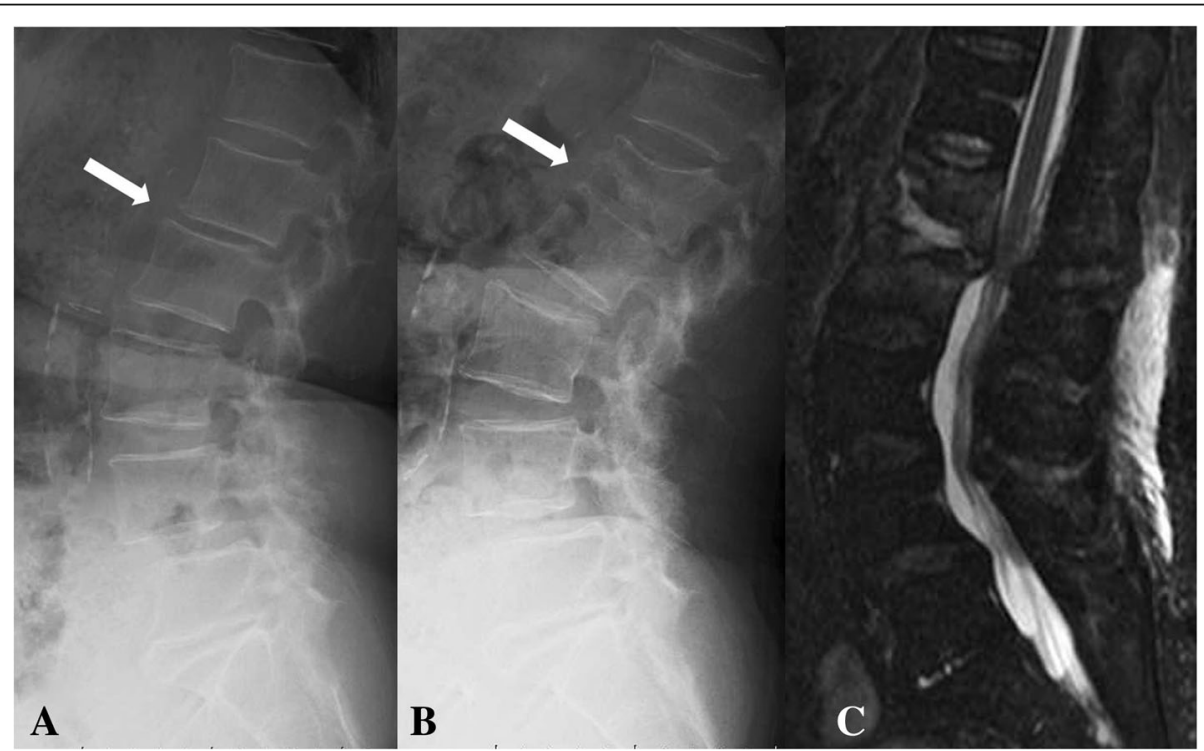

Fig. 1 a Roentgenogram of the lateral lumbar vertebra at the first visit. A slightly narrow L1/2 is observed (white arrows). b Roentgenogram of the lateral lumbar vertebra at 1 month after the first visit. Collapse of L1 and absorbable changes at the caudal side of L1 are observed (white arrows). c T2-weighted fat-suppression magnetic resonance image. Effusion and edema are observed in L1 and L2 


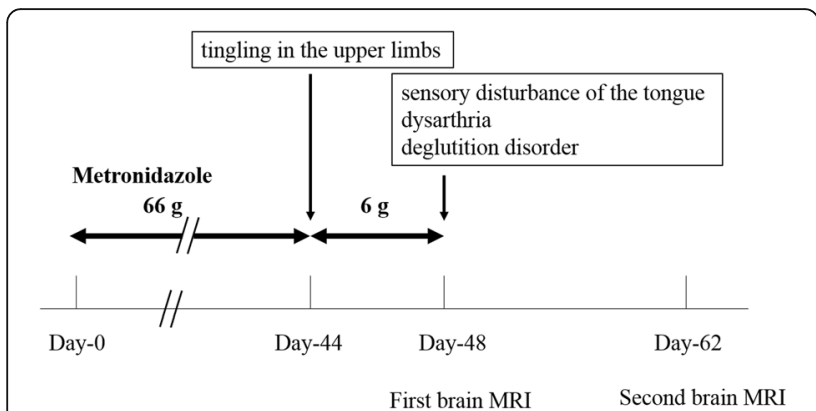

Fig. 2 Time line after the initiation of metronidazole

Cervical spinal disorder was suspected, and cervical spinal MRI demonstrated spinal canal stenosis at C3/4, 5/6, and $6 / 7$. After 4 days, she complained of further symptoms, including sensory disturbance of the tongue, dysarthria, and deglutition disorder. Central nervous system disorder was suspected, and brain MRI was performed. Characteristic brain MRI findings on T2-weighted fluid-attenuated inversion recovery (FLAIR) and diffusion weighted imaging (DWI) led to the diagnosis of MIE (Fig. 3a, b). Metronidazole was discontinued, and her neurological symptoms improved 10 days after discontinuation. On MRI performed 14 days after oral metronidazole discontinuation, most of the abnormal findings of MIE disappeared (Fig. 3c, d). Fortunately, follow-up blood tests revealed the absence of inflammatory reactions 5 days before the discontinuation of metronidazole; no antibiotics were administered after discontinuing metronidazole. At 9 weeks after discontinuation of metronidazole, there was no recurrence of pyogenic spondylitis according to the clinical findings or blood sample results.

\section{Discussion and conclusions}

In the orthopedic field, Staphylococcus aureus is one of the primary bacteria causing bone and joint infections or periprosthetic joint infections [16]. However, reports on infections caused by oral bacteria in the orthopedic field have increased in recent years $[17,18]$. Many kinds of anaerobic bacteria exist in the oral cavity, and Walter et al. [16] described that the diagnosis of anaerobic bone and joint infections was underestimated before molecular identification was established. In cases of anaerobic bone and joint infections, antibiotic treatments included amoxicillin, amoxicillin-clavulanic acid, metronidazole, and clindamycin [19]. Neurotoxicity associated with metronidazole presents with a wide spectrum

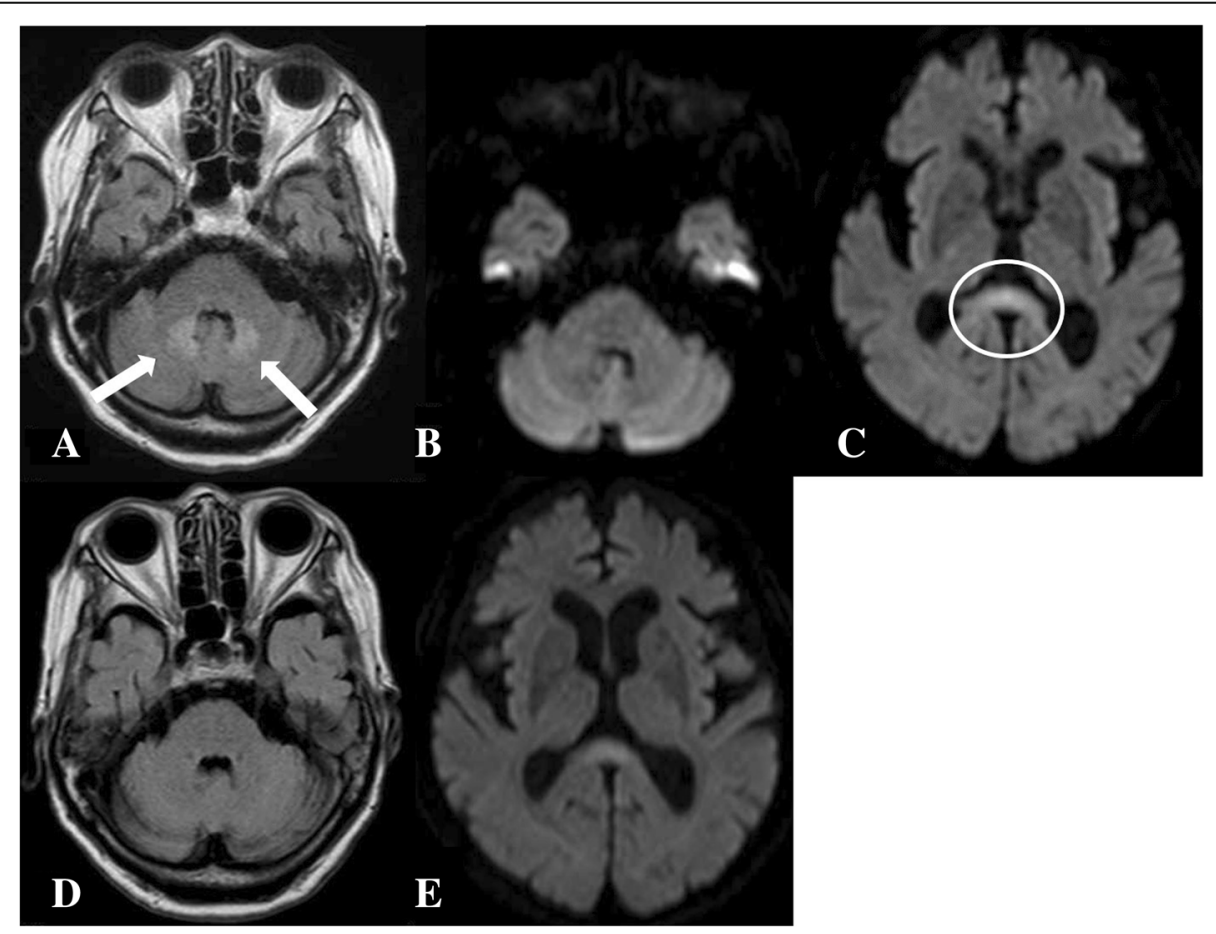

Fig. 3 a Brain magnetic resonance imaging. T2-weighted fluid-attenuated inversion recovery (FLAIR) imaging performed at 4 days after symptom onset shows hyperintense areas in the bilateral basal dentate nuclei (white arrows). b Diffusion-weighted imaging (DWI) performed 4 days after symptom onset showed an almost normal intensity in the bilateral basal dentate nuclei. c DWI performed 4 days after symptom onset revealed a hyperintense T2 signal with compatible cytotoxic edema (white circle). $\mathbf{d}$ At 14 days after discontinuation of oral metronidazole, abnormal findings on T2-weighted FLAIR imaging completely disappeared. e At 14 days after discontinuation of metronidazole, the abnormal findings on DWl almost disappeared 
of symptoms, including mental status changes, peripheral neuropathy, weakness, vertigo, nausea, vomiting, and sensory loss [9, 20]. Osteoarthritis infections caused by Parvimonas micra are rarely encountered by orthopedic surgeons [21]. Therefore, in the current case, sensitivity tests on antibiotics were not performed adequately; thus, adverse effects of metronidazole administration were not adequately recognized. Our literature search yielded no other case reports of MIE occurring during treatment of pyogenic spondylitis. When neurologic symptoms deteriorate during treatment of pyogenic spondylitis with metronidazole, attention should be paid to neurotoxicity, including the possibilities of MIE and peripheral neuropathy caused by metronidazole, in addition to aggravation of pyogenic spondylitis.

However, peripheral neuropathy may appear as a primary symptom, and the diagnosis may be delayed. In the current case, MIE presented as a peripheral neuropathy with a tingling sensation, and the diagnosis of MIE was delayed by 4 days. The proposed mechanism of MIE is thought to involve metronidazole-induced neurotoxicity associated with the oxidation of catecholamine neurotransmitters to semiquinone and nitro anion radicals, which reduce tissue oxygenation and subsequently generate superoxide radicals and hydrogen peroxide. However, the mechanism remains unclear [22].

Metronidazole doses of 45-120 g and treatment durations of 1-12 weeks have been reported to result in MIE [23-25]; however, the doses and durations widely vary. The half-life of metronidazole is 6-8 h; however, the half-life in patients with liver dysfunction or renal dysfunction is approximately 3 -folds higher. Accordingly, it is necessary to limit the total cumulative dose to less than $20 \mathrm{~g}$ [26]. Our patient had mild renal dysfunction, and the total cumulative dose was $66 \mathrm{~g}$. Therefore, when metronidazole is used, the patient's neurological symptoms should be monitored closely [26].

The clinical disease concept of MIE was reported over 20 years ago [3-8]; however, MIE-specific MRI findings have been reported recently [10-15]. The brain MRI findings of MIE were categorized according to the apparent diffusion coefficient, which are as follows: minimal (grade 1) indicating symmetric involvement of one lobe (frontal, temporal, parietal, or occipital) without involvement of the corpus callosum, basal ganglia, thalami, or internal capsules; mild (grade 2) indicating symmetric involvement of two lobes, or of one lobe plus symmetric involvement of one of the corpus callosum, basal ganglia, thalami, or internal capsules; moderate (grade 3 ) indicating symmetric involvement of two lobes plus symmetric involvement of one of the corpus callosum, basal ganglia, thalami, or internal capsules; and severe (grade 4) indicating symmetric, extensive, and confluent involvement of three or all lobes from the ventricular margin to the subcortical white matter, or of two lobes plus symmetric involvement of two of the following: corpus callosum, basal ganglia, thalami, or internal capsules [14, 15].

A direct correlation was found between the high cumulative dose and the lesion severity in the corpus callosum. Moreover, lesions with restricted diffusion and those without restricted diffusion may sometimes have different pathophysiological mechanisms [12]. Previous studies also reported that lesions without restricted diffusion, including those in the cerebellar dentate nucleus, disappeared after metronidazole discontinuation [27, 28]. However, the abovementioned classical finding may or may not be present during the initial stage of the disease [26, 29]. In most cases, MIE is a reversible disease that improves within a few weeks after discontinuation of metronidazole [23, 30]. However, MIE is not always reversible and may result in a fatal outcome $[14,31]$. Therefore, correct and early diagnosis is very important in the management of patients with MIE. Thus, when metronidazole is administered, the dose and duration of treatment should be limited.

In the current case, pyogenic spondylitis was treated with metronidazole for 48 days, and no recurrence was observed for 9 weeks. Although the treatment period exceeded six weeks, which is recommended for treating pyogenic spondylitis [32], the follow-up period was as short as 9 weeks.

The incidence of MIE is not very high, and the majority of patients with MIE recover uneventfully. However, occasionally, the symptoms of peripheral neuropathy persist or become severe. In the current case, there were no sequelae, but the patient was not initially accurately diagnosed. MIE has been well described, but it has been rarely reported in the orthopedic literature. In the past, there are only four cases of possible spondylitis due to Parvimonas micra [21]; however, there may be more potential cases. Orthopedic surgeons should be responsible in improving their knowledge of the appropriate antibiotics for treating infections caused by Parvimonas micra as well as their therapeutic and adverse effects. As orthopedic surgeons are likely to encounter cases of MIE in clinical practice and will therefore need to prescribe metronidazole, it is important that they recognize the condition and its potential complications. With increased awareness, early diagnosis of MRI and discontinuation of metronidazole may become feasible when such patients are referred. Furthermore, we recommend that patients treated with metronidazole should undergo careful and constant surveillance after starting with the medication. 


\section{Abbreviations}

DWl: Diffusion weighted imaging; FLAIR: Fluid-attenuated inversion recovery; MIE: Metronidazole-induced encephalopathy; MRI: Magnetic resonance imaging

\section{Availability of data and materials}

Data are contained within the manuscript.

\section{Authors' contributions}

$\mathrm{KM}, \mathrm{ON}, \mathrm{TK}, \mathrm{DN}$, and TM treated the patient and designed the study. MS and MM collected and analyzed the data and wrote the article. All authors have read and approved the final manuscript.

\section{Ethics approval and consent to participate}

All procedures were part of the standard medical care, and the need for ethics approval and consent to participate was waived.

\section{Consent for publication}

Written informed consent was obtained from the patient for publication of this case report and any accompanying images. A copy of the consent form is available for review by the Editor of this journal.

\section{Competing interests}

The authors declare that they have no competing interests.

\section{Publisher's Note}

Springer Nature remains neutral with regard to jurisdictional claims in published maps and institutional affiliations.

\section{Author details}

'Department of Orthopaedic Surgery, Yanagawa Hospital, 29 Chikushi-machi, Yanagawa 832-0077, Japan. ²Department of Orthopaedic Surgery, Faculty of Medicine, Saga University, 5-1-1 Nabeshima, Saga 849-8501, Japan.

Received: 12 February 2018 Accepted: 6 September 2018

Published online: 18 September 2018

\section{References}

1. Roy U, Panwar A, Pandit A, Das SK, Joshi B. Clinical and neuroradiological spectrum of metronidazole induced encephalopathy: our experience and the review of literature. J Clin Diagn Res. 2016;10:OE01-9.

2. Bottenberg MM, Hegge KA, Eastman DK, Kumar R. Metronidazole-induced encephalopathy: a case report and review of the literature. J Clin Pharmacol. 2011;51:112-6

3. Kusumi RK, Plouffe JF, Wyatt RH, Fass RJ. Central nervous system toxicity associated with metronidazole therapy. Ann Intern Med. 1980;93:59-60.

4. Frytak S, Moertel $\mathrm{CH}$, Childs DS. Neurologic toxicity associated with highdose metronidazole therapy. Ann Intern Med. 1978;88:361-2.

5. Bradley WG, Karlsson IJ, Rassol CG. Metronidazole neuropathy. Br Med J. 1977:2:610-1.

6. Huang YT, Chen LA, Cheng SJ. Metronidazole-induced encephalopathy: case report and review literature. Acta Neurol Taiwanica. 2012;21:74-8.

7. Iqbal SM, Murthy JG, Banerjee PK, Vishwanathan KA. Metronidazole ototoxicity-report of two cases. J Laryngol Otol. 1999:113:355-7.

8. De Bleecker JL, Leroy BP, Meire VI. Reversible visual deficit and corpus callosum lesions due to metronidazole toxicity. Eur Neurol. 2005:53:93-5.

9. Stip E, Darby RR, Bhattacharyya S, Berkowitz AL. Antibiotic-associated encephalopathy. Neurology. 2016:87:1188-9.

10. Kuriyama A, Jackson JL, Doi A, Kamiya T. Metronidazole-induced central nervous system toxicity: a systematic review. Clin Neuropharmacol. 2011;34: 241-7.

11. Cecil KM, Halsted MJ, Schapiro M, Dinopoulos A, Jones BV. Reversible MR imaging and MR spectroscopy abnormalities in association with metronidazole therapy. J Comput Assist Tomogr. 2002;26:948-51.

12. Kim E, Na DG, Kim EY, Kim JH, Son KR, Chang KH. MR imaging of metronidazole-induced encephalopathy: lesion distribution and diffusionweighted imaging findings. AJNR Am J Neuroradiol. 2007:28:1652-8.

13. Hammami N, Drissi C, Sebai R, Araar M, Maatallah Y, Belghith $L$, et al. Reversible metronidazole-induced encephalopathy. J Neuroradiol. 2007;34 $133-6$.
14. McKinney AM, Kieffer SA, Paylor RT, SantaCruz KS, Kendi A, Lucato L. Acute toxic leukoencephalopathy: potential for reversibility clinically and on MR with diffusion-weighted and FLAIR imaging. AJR Am J Roentgenol. 2009; 193:192-206.

15. Heaney CJ, Campeau NG, Lindell EP. MR imaging and diffusion-weighted imaging changes in metronidazole (Flagyl)-induced cerebellar toxicity. AJNR Am J Neuroradiol. 2003:24:1615-7.

16. Fux CA, Wilson S, Stoodley P. Detachment characteristics and oxacillin resistance of Staphylococcus aureus biofilm emboli in an in vitro catheter infection model. J Bacteriol. 2004;186:4486-91.

17. Olsen I, Snorrason F, Lingaas E. Should patients with hip joint prosthesis receive antibiotic prophylaxis before dental treatment? J Oral Microbiol. 2010;2. https://doi.org/10.3402/jom.v2i0.5265.

18. Sonohata M, Kitajima M, Kawano S, Mawatari M. Acute hematogenous infection of revision total hip arthroplasty by oral bacteria in a patient without a history of dental procedures: case report. Open Orthop J. 2014;8:56-9.

19. Walter G, Vernier M, Pinelli PO, Million M, Coulange M, Seng P, et al. Bone and joint infections due to anaerobic bacteria: an analysis of 61 cases and review of the literature. Eur J Clin Microbiol Infect Dis. 2014:33:1355-64.

20. Sonthalia N, Pawar SV, Mohite AR, Jain SS, Surude RG, Rathi PM, et al. Metronidazole-induced encephalopathy in alcoholic liver disease: a diagnostic and therapeutic challenge. J Emerg Med. 2016;51:e79-83.

21. Uemura H, Hayakawa K, Shimada K, Tojo M, Nagamatsu M, Miyoshi-Akiyama T, et al. Parvimonas micra as a causative organism of spondylodiscitis: a report of two cases and a literature review. Int J Infect Dis. 2014:23:53-5.

22. Rao DN, Mason RP. Generation of nitro radical anions of some 5-nitrofurans, 2- and 5-nitroimidazoles by norepinephrine, dopamine, and serotonin. A possible mechanism for neurotoxicity caused by nitroheterocyclic drugs. Jiol Chem. 1987;262:11731-6.

23. Ahmed A, Loes DJ, Bressler EL. Reversible magnetic resonance imaging findings in metronidazole-induced encephalopathy. Neurology. 1995;45: $588-9$.

24. Bahn Y, Kim E, Park C, Park HC. Metronidazole induced encephalopathy in a patient with brain abscess. J Korean Neurosurg Soc. 2010:48:301-4.

25. Lee SS, Cha SH, Lee SY, Song CJ. Reversible inferior colliculus lesion in metronidazole-induced encephalopathy: magnetic resonance findings on diffusion-weighted and fluid attenuated inversion recovery imaging. Comput Assist Tomogr. 2009:33:305-8.

26. Cheong HC, Jeong TG, Cho YB, Yang BJ, Kim TH, Kim HC, et al. Metronidazole-induced encephalopathy in a patient with liver cirrhosis. Korean J Hepatol. 2011;17:157-60.

27. Graves TD, Condon M, Loucaidou M, Perry RJ. Reversible metronidazoleinduced cerebellar toxicity in a multiple transplant recipient. J Neurol Sci. 2009;285:238-40.

28. Seok Jl, Yi H, Song YM, Lee WY. Metronidazole-induced encephalopathy and inferior olivary hypertrophy: lesion analysis with diffusion-weighted imaging and apparent diffusion coefficient maps. Arch Neurol. 2003:60: 1796-800.

29. Bae EH, Ma SK, Kim SK, Kim SW. Metronidazole-induced encephalopathy: a case report. J Nippon Med Sch. 2016;83:44-5.

30. Hwang GH, Sim YJ, Jeong HJ, Kim GC, Sin BW, Jung JH. Metronidazole induced encephalopathy with peripheral polyneuropathy in patient with spinal cord injury. Korean J Spine. 2012;9:44-8.

31. Groothoff MV, Hofmeijer J, Sikma MA, Meulenbelt J. Irreversible encephalopathy after treatment with high-dose intravenous metronidazole. Clin Ther. 2010;32:60-4.

32. Berbari EF, Kanj SS, Kowalski TJ, Darouiche RO, Widmer AF, Schmitt SK, et al. 2015 Infectious Diseases Society of America (IDSA) clinical practice guidelines for the diagnosis and treatment of native vertebral osteomyelitis in adults. Clin Infect Dis. 2015:61:e26-46. 